\title{
Impact of Helicobacter pylori-related Microbial Dysbiosis in the Pathogenesis of Metabolic Syndrome and Gastrointestinal Dysmotility Disorders
}

TO THE EDITOR: Singh et al $^{1}$ reviewed data on gut dysbiosis and/or interactions involved in the pathogenesis of gastrointestinal tract (GIT) dysmotility and metabolic syndrome (MetS) conditions including irritable bowel syndrome (IBS), functional dyspepsia (FD), and type 2 diabetes mellitus (T2DM). They concluded that MetS is greatly influenced by the gut, thereby metabolic disorders could begin there.

In this respect, Helicobacter pylori infection, a global high burden, induces GIT dysmotility (mainly abnormal gastric emptying). ${ }^{2}$ Likewise, $H$. pylori-associated MetS conditions are related with dysmotility-stimulated gastrointestinal microbial overgrowth, potentially leading to bacteremia with systemic conditions. ${ }^{3}$ As mentioned by the authors, ${ }^{1}$ MetS occurs commonly in patients with small intestinal bacterial overgrowth (SIBO). ${ }^{3}$ Gut microbiota has been connected with MetS parameters, and $H$. pylori-related T2DM, a parameter of MetS, provokes diabetic gastroparesis which may contribute to hepatic encephalopathy (HE) by altering GIT motility and inducing SIBO and subsequent bacterial translocation. ${ }^{4} \mathrm{H}$. pylori-associated insulin resistance (IR), the main MetS key component, ${ }^{5}$ may further provoke diabetic gastroparesis, and IR-associated diabetes is linked with bacteremia and sepsis triggering subsequent systemic conditions. In this respect, dysbiosis of the GIT microbiome, including oral and gastric $H$. pylori bacteria and several non- $H$. pylori microorganisms, is related with systemic inflammatory processes and conditions, including HE. In the setting of defensins, also mentioned by the authors, ${ }^{1}$ the upregulation of human $\beta$ defensin-1 (hBD-1) may present a biomarker of bacterial translocation involved in the pathogenesis of $\mathrm{HE}^{6}{ }^{6}$ Moreover, H. pylori infection appears to induce hBD-1 mRNA expression, ${ }^{6}$ which also may contribute to the pathophysiology of the central nervous system pathologies, by altering both innate and adaptive immune system reactions. ${ }^{7}$

Regarding H. pylori and MetS-related FD accompanied by GIT dysbiosis, $H$. pylori eradication may reduce the risk of MetS criteria in FD patients. ${ }^{8}$ Likewise, H. pylori and MetS-related IBS may be associated with dysbiosis, and $H$. pylori eradication therapy may decrease the risk of IBS. Interestingly, $H$. pylori infection and MetS-related dysbiosis may represent major risk indicators of Parkinson's disease (PD). ${ }^{5}$ In this respect, $H$. pylori infection may affect the bioavailability of L-dopa, a dopamine precursor used for therapy of PD, by injuring the duodenal mucosa, the main place of L-dopa main absorption. On the other hand, H. pylori eradication seems to improve L-dopa onset plus duration time, and PD's GIT motor fluctuations. Besides, MetS and H. pylori infection are connected with systemic sclerosis (SSc). ${ }^{9} \mathrm{SSc}$, by inducing GIT dysmotility and SIBO, may trigger the development of complications with high morbidity/mortality. Moreover, SSc is associated with multiple sclerosis, the latter of which is also connected with dysbiosis. Eradication of H. pylori infection may influence the pathophysiology of SSc and multiple sclerosis, at least in certain populations. ${ }^{10}$ Important to note, however, is that the relationship between $H$. pylori infection and the aforementioned chronic diseases has not been established yet, and, thus, further large-scale studies are needed.

\footnotetext{
Jannis Kountouras, ${ }^{1 *}$ Apostolis Papaefthymiou, ${ }^{1,2,3}$ Stergios A Polyzos, ${ }^{3}$ Elisabeth Vardaka, ${ }^{1,4}$ Marina Boziki, ${ }^{5}$ Anthony Kyriakopoulos, ${ }^{6}$ Fotios Sampsonas, ${ }^{7}$ George Agrotis, ${ }^{8}$ Kyriaki Karafyllidou, ${ }^{9}$ and Michael Doulberis ${ }^{1,3,10}$

${ }^{1}$ Department of Medicine, Second Medical Clinic, Aristotle University
} 
of Thessaloniki, Ippokration Hospital, Thessaloniki, Macedonia, Greece; ${ }^{2}$ Department of Gastroenterology, University Hospital of Larisa, Mezourlo, Larisa, Greece; ${ }^{3}$ First Laboratory of Pharmacology, Aristotle University of Thessaloniki, Thessaloniki, Macedonia, Greece; ${ }^{4}$ Department of Nutritional Sciences and Dietetics, School of Health Sciences, International Hellenic University, Alexander Campus, Thessaloniki, Macedonia, Greece; ${ }^{5}$ Second Neurological Department, Aristotle University of Thessaloniki, AHEPA University General Hospital of Thessaloniki, Thessaloniki, Macedonia, Greece; ${ }^{6}$ Department of Research and Development, Nasco AD Biotechnology Laboratory, Piraeus, Greece; ${ }^{7}$ Department of Respiratory Medicine, University Hospital of Patra, Rion, Patra, Greece; ${ }^{8}$ Department of Radiology, University Hospital of Larisa, Mezourlo, Larisa, Greece; ${ }^{9}$ Department of Pediatrics, University Children's Hospital of Zurich, Zurich, Switzerland; and ${ }^{10}$ Division of Gastroenterology and Hepatology, Medical University Department, Kantonsspital Aarau, Aarau, Switzerland

1. Singh R, Zogg H, Wei L, et al. Gut microbial dysbiosis in the pathogenesis of gastrointestinal dysmotility and metabolic disorders. J Neurogastroenterol Motil 2021;27:19-34.

2. Gümürdülü $\mathrm{Y}$, Serin $\mathrm{E}$, Ozer B, et al. The impact of $\mathrm{B}_{12}$ treatment on gastric emptying time in patients with Helicobacter pylori infection. J Clin Gastroenterol 2003;37:230-233.

3. Fialho A, Fialho A, Thota P, McCullough AJ, Shen B. Small intestinal bacterial overgrowth is associated with non-alcoholic fatty liver disease. J Gastrointestin Liver Dis 2016;25:159-165.

4. Ampuero J, Ranchal I, del Mar Díaz-Herrero M, del Campo JA, Bautista JD, Romero-Gómez M. Role of diabetes mellitus on hepatic encephalopathy. Metab Brain Dis 2013;28:277-279.

5. Franceschi F, Gasbarrini A, Polyzos SA, Kountouras J. Extragastric diseases and Helicobacter pylori. Helicobacter 2015;20(suppl 1):40-46.

6. Kountouras J, Zavos C, Polyzos SA, Deretzi G. Potential impact of Helicobacter pylori-related human beta-defensin-1 on hepatic encephalopathy and neurodegeneration. Ann Gastroenterol 2016;29:99.

7. Kountouras J, Deretzi G, Gavalas E, et al. A proposed role of human defensins in Helicobacter pylori-related neurodegenerative disorders. Med Hypotheses 2014;82:368-373

8. Kountouras J, Gavalas E, Papaefthymiou A, et al. Trimebutine maleate monotherapy for functional dyspepsia: a multicenter, randomized, double-blind placebo controlled prospective trial. Medicina 2020;56:339.

9. Radićc M, Kaliterna DM, Bonacin D, et al. Is Helicobacter pylori infection a risk factor for disease severity in systemic sclerosis? Rheumatol Int 2013;33:2943-2948.

10. Kountouras J, Zavos C, Gavalas E, et al. Helicobacter pylori may be a common denominator associated with systemic and multiple sclerosis. Joint Bone Spine 2011;78:222-223, author reply 223.

Financial support: None.

Conflicts of interest: None.

Author contributions: Jannis Kountouras had the idea and drafted the article; and Apostolis Papaefthymiou, Stergios A Polyzos, Elisabeth Vardaka, Marina Boziki, Anthony Kyriakopoulos, Fotios Sampsonas, George Agrotis, Kyriaki Karafyllidou, and Michael Doulberis have read and agreed with the final manuscript. 